\title{
Synthesis of SBA-1 Mesoporous Silica Crystals with Tunable Pore Size Using Sodium Silicate and Alkyltrimethylammonium Surfactants
}

\author{
Man-Chien Chao, Hong-Ping Lin, ${ }^{\dagger \dagger}$ Da-Shin Wang, ${ }^{\dagger}$ and Chung-Yuan Mou \\ Department of Chemistry, National Taiwan University, Taipei, 106 Taiwan \\ ${ }^{\dagger}$ Department of Chemistry, National Cheng Kung University, Tainan, 701 Taiwan
}

(Received January 5, 2004; CL-040004)

The spherical micelle-templated SBA-1 mesoporous silicas with different pore size were conveniently synthesized from commercially available alkyltrimethylammonium surfactants and sodium silicate components in dilute solution.

The periodic 3-D SBA-1 mesoporous silicas with controllable crystal morphologies have been receiving much attention because of their uses in catalyst supports, separation, waveguides, and optical devices. ${ }^{1,2}$ Basically, the SBA-1 could be considered as spherical micelle-templated mesoporous silica well-packed in a cubic $\operatorname{Pm} \overline{3} n$ mesostructure. To maintain the high-curvature spherical building block, using large head-group alkyltriethylammonium surfactant as template, addition of spherical-micelle stabilizing agent or careful control on the acidity of the reaction solution has been proposed. ${ }^{3-5}$ According to the theory of surfactant, ${ }^{6}$ surfactants of the lyotropic behavior tend to aggregate into spherical micelles in dilute solution. Consequently, we introduced a new concept to synthesize the SBA-1 mesoporous silica in dilute solution. At a properly low concentration of surfactant, the well-ordered SBA-1 mesoporous silicas in crystal form have been synthesized by using the commercially available alkyltrimethylammonium surfactants with relatively small head group at $\mathrm{pH}$ value of 2.0, and the pore size and morphology of the SBA-1 mesoporous silica crystal change with the surfactant chain length.

Synthetic procedure and chemical components of the mesoporous silica SBA-1 in dilute solution were described as followed: the acidified sodium silicate solution as silica source was obtained by pouring a diluted sodium silicate aqueous solution (sodium silicate: $\mathrm{H}_{2} \mathrm{O}$ by weight $=1: 50$ ) to a $1.2 \mathrm{M} \mathrm{H}_{2} \mathrm{SO}_{4}$ solution. This acidified sodium silicate solution was mixed with an alkyltrimethylammonium surfactants $\left(\mathrm{C}_{n}\right.$ TMAX, $n=14-18$; $\mathrm{X}=\mathrm{Cl}$ or $\mathrm{Br}$, Acrôs or TCI) solution. The final $\mathrm{pH}$ value was adjusted to 2.0. The mixture was stirred for $5 \mathrm{~min}$ and then the reaction was continued under a static condition at $30^{\circ} \mathrm{C}$ for $2-$ 4 days. The resultant white precipitates were filtered, washed with distilled water and dried at $60^{\circ} \mathrm{C}$. The as-synthesized samples were calcined in air at $560^{\circ} \mathrm{C}$ for $6 \mathrm{~h}$ to remove the organic templates. The molar composition of the reaction mixture was: 1.0 $\mathrm{C}_{n}$ TMAX:2.15 $\mathrm{SiO}_{2}: 1.67 \mathrm{NaOH}:(4.35-8.90) \mathrm{H}_{2} \mathrm{SO}_{4}:(926-$ 5556) $\mathrm{H}_{2} \mathrm{O}$.

Figure 1 shows the powder X-ray diffraction (XRD) patterns of the calcined mesoporous silica prepared with the $\mathrm{C}_{n}$ TMAX surfactants of $n=14-18$, respectively. One can clearly see that all mesoporous silicas have three well-resolved sharp diffraction peaks at low angle range of 1.5-3.0, which are indexed to 200, 210, 211 reflections based on the $P m \overline{3} n$ SBA- 1 cubic mesostructure. Additional peaks in the range of $2 \theta=3.0-6.0^{\circ}$ indicate a high degree of cubic mesostructural organization. Moreover, all samples represent typical type IV $\mathrm{N}_{2}$ adsorption-desorption isotherms with sharp capillary condensation at $P / P_{0}$ within $0.2-0.4$. In Table 1, one can find that the unit cell and pore size of SBA-1 samples increase with the chain length of the surfactant. ${ }^{7}$ These samples all possess the mesoporous silica advantages of the high adsorption volume of $300-500 \mathrm{~cm}^{3} / \mathrm{g}$ in STP and large surface area of about $1000 \mathrm{~m}^{2} / \mathrm{g}$.

Table 1. Structural properties of SBA-1 mesoporous silica ${ }^{a}$ with the surfactant of different chain length

\begin{tabular}{ccccc}
\hline & $d_{210} / \mathrm{nm}$ & $S_{\mathrm{BET}} / \mathrm{m}^{2} / \mathrm{g}$ & $V_{\mathrm{p}} / \mathrm{cm}^{3} / \mathrm{g}$ & $W_{\mathrm{BJH}} / \mathrm{nm}$ \\
\hline $\mathrm{C}_{14} \mathrm{TMABr}$ & 3.6 & 1039 & 0.67 & 1.8 \\
$\mathrm{C}_{16} \mathrm{TMABr}$ & 3.9 & 1217 & 0.71 & 2.1 \\
$\mathrm{C}_{18} \mathrm{TMACl}$ & 4.5 & 1198 & 0.88 & 2.6 \\
\hline
\end{tabular}

${ }^{\mathrm{a}} S_{\mathrm{BET}}$, the BET specific surface area, $V_{\mathrm{p}}$, the primary mesopore volume; $W_{\mathrm{BJH}}$, the pore diameter calculated using the BJH method.

Because the self-assembling capability of the surfactant depends on both the surfactant's hydrophobicity (i.e. chain length) and the concentration, ${ }^{6}$ a fine control on the concentration of the surfactant is essentially important for preparing high-quality SBA-1 mesoporous silica. To maintain the sphere-micelle template, we found a suitable concentration for all these $\mathrm{C}_{n}$ TMAX surfactant is $0.060-0.010 \mathrm{M}$. At surfactant concentration lower than $0.010 \mathrm{M}$, the mesostructure of C14TMAB-templated mesoporous silica is disordered because of the shorter chain length of $\mathrm{C}_{14} \mathrm{TMABr}$ surfactants. In contrast, the longer chain-length $\mathrm{C}_{18} \mathrm{TMACl}$ and $\mathrm{C}_{16} \mathrm{TMABr}$ surfactants tend to induce a phase transformation from cubic $P m \overline{3} n$ to hexagonal $p 6 \mathrm{~mm}$ structure at surfactant concentration higher than $0.06 \mathrm{M}$.
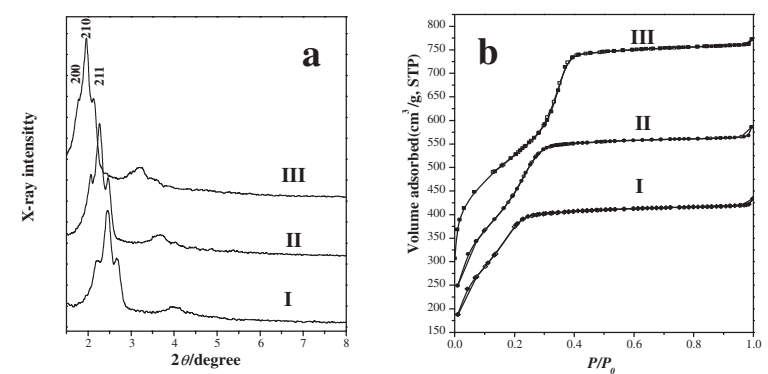

Figure 1. The XRD patterns (a) and $\mathrm{N}_{2}$ adsorption-desorption isotherms (b) of the calcined mesoporous silica synthesized from $\mathrm{C}_{n}$ TMAX-sodium silicate- $\mathrm{H}_{2} \mathrm{O}$ components $\left(\left[\mathrm{C}_{n}\right.\right.$ TMAX $=$ $0.020 \mathrm{M}])$ at $30^{\circ} \mathrm{C}$ in aqueous solutions of $\mathrm{pH} \approx 2.0$. Sample I by $\mathrm{C}_{14} \mathrm{TMABr}$; Sample II by $\mathrm{C}_{16} \mathrm{TMABr}$; Sample III by $\mathrm{C}_{18}$ TMACl. 
Besides of the maintenance of the spherical-micelle structure, we found the common feature on SBA-1 synthesis is the relatively slow self-assembling rate. Generally, the reaction time for SBA-1 is about hours to days longer than that for the MCM41 or SBA-3 mesostructure (only a few minutes). Thus, a lowtemperature condition and hydrolysis-needed TEOS or silanes were performed in many previous reports. ${ }^{8,9}$ In our synthetic method, we combine the kinetic idea and silica chemistry to achieve the criteria for SBA-1 mesoporous silica formation by empolying a low-concentration reaction solution and adjusting the reaction solution at $\mathrm{pH}$ around 2.0 (isoelectric point of silica; IEP), where the silica condensation is slowest. ${ }^{10}$ Consequently, the cheap sodium silicate can be used as silica source instead of TEOS or silanes.

In addition to the synthesis of SBA-1 mesoporous silica, the dilute synthetic condition can slow down the self-assembling rate of surfactant and silica species to provide a time long enough for higher-level assemble of micron-crystals. ${ }^{11}$ In general, when the surfactant concentration at $0.020 \mathrm{M}$, we found that the $\mathrm{C}_{n}$ TMAX-templated SBA-1 mesoporous silica turns to crystal form. From the low-magnification SEM observations, the homogeneity of each sample is high. Figures $2 \mathrm{a}-\mathrm{c}$ display the representative SEM images of the SBA-1 mesoporous silica prepared with the different alkyltrimethylammonium surfactants. Obviously, the micron-sized crystal shape varied with the surfactant chain length. The $\mathrm{C}_{18}$ TMACl-templated SBA-1 crystal is in the cube form, and the face is assigned to Miller index (100) in the cubic structure. The $\mathrm{C}_{16} \mathrm{TMABr}$-templated crystal is in cube truncated by the hexagons, and the square and hexagonal faces are defined as (100) and (110) planes, respectively. The $\mathrm{C}_{14} \mathrm{TMABr}$-templated crystal is in polyhedral form. In contrast, when the surfactant concentration is higher that $0.05 \mathrm{M}$, the self-assembling rate of surfactant and silica species would be too fast to offer a proper time for crystal growth. As expected, the SBA-1 mesoporous silica is in an isotropicallygrowing spherical form without apparent crystal facet (Figure 2d). Accordingly, besides of the $\mathrm{pH}^{12}$ the concentration and
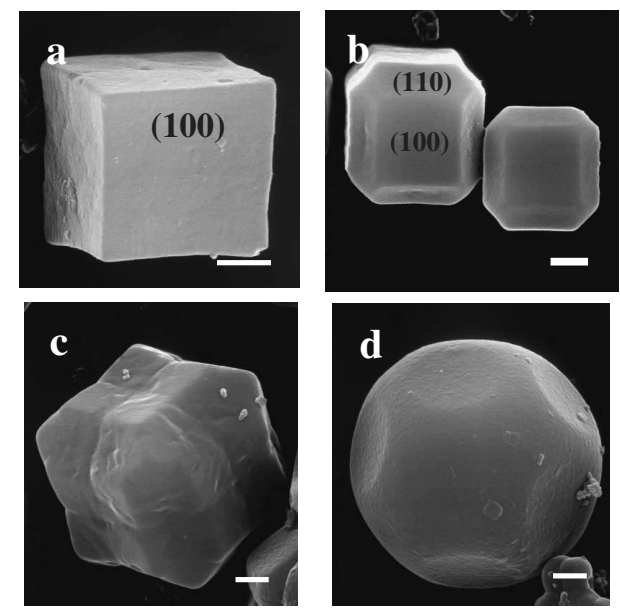

Figure 2. The high-magnification SEM images of the representative SBA-1 mesoporous silica crystals of $\mathrm{C}_{n}$ TMAX.-templated SBA-1 samples of Figure 1. (a) Sample III by $\mathrm{C}_{18}$ TMACl; (b) Sample II by $\mathrm{C}_{16} \mathrm{TMABr}$; (c) Sample I by $\mathrm{C}_{14} \mathrm{TMABr}$. (d) The SBA-1 mesoporous silica prepared from $\mathrm{C}_{18} \mathrm{TMACl}$ at $\left[\mathrm{C}_{18} \mathrm{TMACl}\right]=0.05 \mathrm{M}$. The scale bar $=1.0 \mu \mathrm{m}$. chain length of the surfactant also have apparent effect on the SBA-1crystal morphology.

To explain the effect of surfactant chain length on the crystal shape, it would be reasonably supposed that the crystal morphology of SBA-1 crystals can be changed in the presence of certain $\mathrm{C}_{n}$ TMAX surfactants. ${ }^{11}$ According to the result of SEM observations, the $\mathrm{C}_{18} \mathrm{TMACl}$ surfactants of large hydrophobicity prefer to have a strong interaction with silica on (100) face. ${ }^{13}$ The energetically stable (100) face dominates the final cube form. For the $\mathrm{C}_{16} \mathrm{TMABr}$, the interaction-strength difference between (100) and (110) faces are not great, and then the (100) and (110) faces can coexist on the cube truncated by hexagons. While, the $\mathrm{C}_{14} \mathrm{TMABr}$ has the less hydrophobicity, the interaction strength between $\mathrm{C}_{14} \mathrm{TMABr}$ and silica on the possible faces of the cubic structure is very close. Thus, the polyhedral crystals are formed. However, the formation mechanism of the SBA-1 mesoporous silica crystals is extremely complicated, further studies on the formation mechanism and other experimental factors (e.g. surfactant/silica ratio, acid source, $\mathrm{pH}$ value, addition of salts and cosurfactants and temperature) are necessary for manipulating the crystal shape.

In conclusion, with a proper control on the surfactant concentration, the SBA-1 mesoporous silica with tunable pore size of $1.8-2.6 \mathrm{~nm}$ and in different crystal shapes can be obtained by using cheapest silica source (i.e. sodium silicate) and commercially available alkyltrimethylammonium surfactants at ambient temperature. This convenient synthetic method and cheap chemical components would make the 3 -D cubic SBA-1 mesoporous silica crystal to be extensively investigated on its versatile applications for catalyst, solid templates, and absorbents.

This research was financially supported by National Science Council of Taiwan (NSC92-2113-M-006-015).

\section{References}

1 M. Kaneda, T. Tsubakiyama, A. Carlsson, Y. Sakamoto, T. Ohsuna, and O. Terasaki, J. Phys. Chem. B, 106, 1256 (2002).

2 S. Che, Y. Sakamoto, O. Terasaki, and T. Tatsumi, Chem. Lett., 2002, 214.

3 M. P. Kapper and S. Inagaki, Chem. Mater., 14, 3509 (2002).

4 S. Che, S. Lim, M. Kaneda, H. Yoshitake, O. Terasaki, and T. Tatsumi, J. Am. Chem. Soc., 124, 13962 (2002).

5 S. Guan, S. Inagaki, T. Ohsuna, and O. Terasaki, J. Am. Chem. Soc., 122, 5660 (2000).

6 K. Holmberg, B. Jönsson, B. Kronberg, and B. Lindman, "Surfactants and Polymers in Aqueous Solution," Wiley, England (2002).

7 a) M. J. Kim and R. Ryoo, Chem. Mater., 11, 487 (1999). b) H. P. Lin, S. Cheng, and C. Y. Mou, J. Chin. Chem. Soc., 43, 375 (1996).

8 S. Inagaki, S. Guan, Y. Fukushima, T. Ohsuna, and O. Terasaki, J. Am. Chem. Soc., 121, 9611 (1999).

9 M. C. Liu, H. S. Sheu, and S. Cheng, Chem. Commun., 2002, 2854.

10 R. K. Iler, "The Chemistry of Silica: Solubility, Polymerization, Colloid and Surface Properties, and Biochemistry," John Wiley \& Sons, New York (1979).

11 S. Mann, "Biomineralization Principles and Concepts in Bioinorganic Materials Chemistry," Oxford University Press, New York (2001).

12 M. C. Chao, H. P. Lin, D. S. Wang, and C. Y. Mou, J. Mater. Chem., 13, 2853 (2003).

13 H. P. Lin and C. Y. Mou, Acc. Chem. Res., 35, 927 (2002). 